\title{
Prevalence of Hydatid Cyst in Cattle at Municipal Abbatoir of Shire \\ Asmerom Asfaw ${ }^{1}$ and Berihun Afera ${ }^{2 *}$
}

${ }^{1}$ Tigray Bureau of agriculture and Rural Development, Ethiopia

${ }^{2}$ College of Veterinary Medicine, Mekelle University, Ethiopia

\begin{abstract}
Cross sectional study with the objective of assessing the prevalence of hydatid cyst on 440 cattle was conducted from May to June 2014 in Shire municipal abattoir. The present finding revealed that the overall rate of the parasite was $32 \%$. Sex wise distribution of the parasite was $-29.9 \%$ and $3.2 \%$ in male and female cattle with no statistical significance variation between male and female. Similarly, the rates in adult and old cattle were $23.6 \%$ and $8.4 \%$ respectively. The rate of the parasite in adult and old cattle showed significance variation $(p<0.05)$. Similarly the prevalence in local and cross breed was also $31.6 \%$ and $0.5 \%$ with no statistical variation between the two breeds. Moreover, hydatid cysts prevalence of each organ at the abattoir survey indicated 70 (17.5\%) in lung; 65 (14.77\%) in liver; $2(0.45 \%)$ in heart and $4(0.9 \%)$ in kidney respectively. This indicates that the parasite was found in cattle of the area and it was responsible for high condemnation of different organs. Hence, strategic deworming with strong surveillance system and good management practice of animals is essential for reduction of the disease prevalence.
\end{abstract}

Keywords: Abattoir; Cattle; Hydatid cyst; Prevalence; Shire

\section{Introduction}

In Ethiopia, meat inspection was started in 1910's with the aim of improving productivity and trade in animals and animal products as well as protecting the public from zoonotic diseases. Despite one century of experience on modern animal health activities, there exist very few legislation and regulation animal diseases control and meat inspection [1].

Hydatidosis is one of the major parasitic problems of domestic animals and zoonotic diseases that cause considerable economic losses and public health problems worldwide [2]. Ecchinococcosis is a zoonotic infection caused by adult or larval (metacestode) stages of cestodes belonging to the genus Echinococcus and the family Taeniidae. Hydatidosis/Cystic Ecchinococcosis, also known as Hydatid Disease, is an infection caused by the larval stage of the flatworm Echinococcus granulosus. It has a cosmopolitan distribution and is one of the most widespread parasitic zoonoses. Hydatidosis is of importance since humans serve as incidental intermediate [3].

This multi host parasite is prevalent all over the world and annually the economic loss in livestock due to this parasite is significant. In Africa, hydatid disease is reported more common in cattle those are communally owned or are raised of free range, and which associated more intimately with domestic dogs. Hydatidosis in domestic ruminants inflicts enormous economic damage due to the condemnation of the affected organs and lowering of the meat, milk and wool production [4].

Factors governing the prevalence of hydatidosis are given locally being associated with prevailing specific social, cultural, environmental and epidemiological situations. Human behaviour plays a significant role in the epidemiology and the dynamic of transmission differ between the dog and its normal intermediate and human hosts $[5,6]$. The outcome of the infection in livestock is hydatid cyst development in the lung, liver, spleen, kidney etc. [7].

In Ethiopia, hydatidosis has been known and documented as early as 1970 's. Hydatidosis is the major cause of organ condemnation in most Ethiopian abattoirs and slaughter houses causing huge economic losses [8]. In Ethiopia, studies conducted in different abattoirs indicated that cystic hydatidosis is prevalent and considerable economic losses are associated with it. Certain deeply rooted traditional activities could be commonly described as factors substantiating the spread and high prevalence rates of the diseases in some areas of the country. These may include the widespread backyard animal slaughtering practice, the corresponding absence of rigorous meat inspection procedures and long standing habit of the most Ethiopian people to feed their dogs with condemned offal which in effect, facilitate the maintenance of the perfect life cycle of E.granulosus and consequently high rate of infection of susceptible hosts [9].

In Tigray, particularly in North West zone, livestock rising is an important activity from which food and non-food commodities are produced. Livestock plays a key role in the daily life of the population; particularly, the peasant owns over $95 \%$ of the country. But the productivity remains very low due to lack of disease control and poor husbandry practice. And previously there was no any data on the study area to determine the prevalence of the disease and its rate in different organs. Hence, the objective of this paper was to determine the prevalence of bovine hydatidosis at Shire municipal abattoir.

\section{Materials and Methods}

\section{Study area}

The study was conducted from May 2014 to June, 2014 in Shire, North West Zone, at shire municipal abattoir in Tigray regional state located in the northern Ethiopia. Shire is $1087 \mathrm{~km}$ far away from Addis Ababa with an altitude of 1600-2200 above sea levels, the rain fall gets in summer season $700-1135 \mathrm{~mm}(877.6 \mathrm{~mm})$, the temperature ranges 18 $34.6^{\circ} \mathrm{c}$. Livestock population of the woreda has a total head of 116092 of cattle, 42567 sheep, 10577 of goats, 8799 of donkeys \& horse, 95 of mules, 428 of camel, 14832 of chickens and 9714 honey bee colonies. The area was covered by different trees, shrubs, herbs, and grasses.

*Corresponding author: Berihun Afera, College of Veterinary Medicine, Mekelle University, Ethiopia, Tel: +251-910-276541; E-mail: berihun414@yahoo.com

Received July 09, 2014; Accepted July 28, 2014; Published July 30, 2014

Citation: Asfaw A, Afera B (2014) Prevalence of Hydatid Cyst in Cattle at Municipal Abbatoir of Shire. J Veterinar Sci Technol 5: 186. doi:10.4172/2157-7579.1000186

Copyright: ( 2014 Asfaw A, et al. This is an open-access article distributed under the terms of the Creative Commons Attribution License, which permits unrestricted use, distribution, and reproduction in any medium, provided the original author and source are credited. 


\section{Study population}

The study animals comprise indigenous zebu cattle slaughter at shire municipal abattoir. These cattle presented for slaughtering was local breeds.

\section{Study design}

A cross sectional abattoir survey was conducted on randomly selected cattle and for the examination of the cyst ante-mortem \& postmortem inspection procedures were carried.

\section{Sample size}

Since there is no study conducted previously about the prevalence \& economic importance in Shire abattoir the expected prevalence of $50 \%$ using $95 \%$ level of confidence and $5 \%$ absolute precision was used according to the formula of Thrustfield [10].

$$
\begin{aligned}
& \mathrm{N}=\frac{\left.1.96^{2} \times \text { per(1-pex }\right)}{\mathrm{d}^{2}} \\
& \text { Where, } \mathrm{n}=\text { required sample size } \\
& \mathrm{P} \exp =\text { expected prevalence } \\
& \mathrm{d}=\text { desired absolute precision }
\end{aligned}
$$

Hence a total of 384 animals were selected for inspection purpose but to increase the precision of the result 440 head of cattle were examined during the study period.

\section{Data collection}

Antemortem inspection: Ante-mortem inspection was carried out using visual examination to evaluate the body condition of the animals and interview was also conducted to obtain data on animal's origin.

Post-mortem inspection: Following a thorough visual inspection, palpation and incision of some suspected organs (such as liver and lung) [4]. All the hydatid cysts found in these organs were collected to conduct the due to cyst infection is registered.

Data analysis: The data was entered in to Microsoft excel spread sheet and coded properly and the coded data were transferred to SPSS version 16. For data analysis descriptive statistics were used and significant association was considered when $\mathrm{p}$ value was less than 0.05 .

\section{Results}

A total of 440 cattle were examined and the overall prevalence of the parasite was $32 \%(141 / 440)$. And the age wise distribution of hydatidosis was higher in adult (23.6\%) than old aged cattle $(8.4 \%)$ as indicated in (Table1).

The rate of the disease in different breed of cattle showed that high

\begin{tabular}{|c|c|c|c|c|}
\hline Age & Total animals & No of positive & $\mathbf{X}^{2}$ & p-value \\
\hline Adult & 291 & $104(23.6 \%)$ & 5.383 & 0.020 \\
\hline Old & 149 & $37(8.4 \%)$ & & \\
\hline Total & 440 & $141(32 \%)$ & & \\
\hline
\end{tabular}

Table 1: Prevalence of bovine hydatidosis in age group.

\begin{tabular}{|c|c|c|c|c|}
\hline Breed & Total animals slaughtered & No of positive & $\mathrm{X}^{2}$ & $\mathrm{p}$-value \\
\hline Local & 432 & $139(31.6 \%)$ & 0.186 & 0.666 \\
\hline Cross & 8 & $2(0.5 \%)$ & & \\
\hline Total & 440 & $141(32 \%)$ & & \\
\hline
\end{tabular}

Table 2: The type of animal breed slaughtered at shire municipal abattoir.

\begin{tabular}{|c|c|c|c|c|}
\hline Sex & Total animal & No of positive & $\mathrm{X}^{2}$ & p-value \\
\hline Female & 45 & $14(3.2 \%)$ & 0.20 & 0.887 \\
\hline Male & 395 & $127(29.9 \%)$ & & \\
\hline Total & 440 & $141(32 \%)$ & & \\
\hline
\end{tabular}

Table 3: The type of animal based on sex.

\begin{tabular}{|c|c|c|}
\hline organ & Total no of condemned organ & Percentage \\
\hline Lung & 70 & $(15.9 \%)$ \\
\hline Liver & 65 & $(14.8 \%)$ \\
\hline Kidney & 4 & $(0.9 \%)$ \\
\hline Heart & 2 & $(0.5 \%)$ \\
\hline Total & 141 & $(32.1 \%)$ \\
\hline
\end{tabular}

Table 4: Organ wise cyst distribution and price of each condemned organ.

rate of the hydatid cyst was found in local breeds compared to that of cross breed cattle as indicated in (Table 2).

Sex wise distribution of the cyst also indicated that high rate of hydatidosis in male was recorded as shown in (Table 3). The distribution of hydatidosis in different organs was $15.9 \%$ in lung followed by liver (14.8\%), kidney (0.9\%), and heart $(0.5 \%)$. as indicated in (Table 4$)$.

\section{Discussion}

In the current finding the prevalence of hydatidosis in cattle was $32 \%$ which was higher than with the report of Dawit [11] who indicated prevalence of $28.09 \%$ in cattle in Mekelle abattoir. The difference may be due to variation in the agro ecology of the study areas and the origin of the animals in the study areas. But compared to the finding of Feseha [12] with the rate of $55.6 \%$ in Debre-zeit the present finding was lower. However the present finding was higher compared to the study by Feseha [12], who reported the rate of hydatidosis $24.3 \%$, and $9.4 \%$ in Gondar and Diredawa respectively.

The high rate of the parasite in the current finding might be due to the greater number of stray dogs as well as wild carnivores. These and other socioeconomic realities in the study area are responsible for further propagation of hydatid disease. In addition, backyard slaughtering of ruminants might be also responsible for dissemination of the parasite.

The peculiar geographic feature of shire due to the presence of Tekeze River and bushy areas along the riversides is an ideal home for many foxes and hyenas. Hence, besides domestic dogs these wild carnivores play a significant role in maintenance of the life cycle and become potential source of infection for domestic ungulates.

In general terms, throughout the world, there had been different magnitude records of hydatidosis in cattle with low, medium and high rates of occurrences. Generally the variation in prevalence rate among different geographical locations could be attributed to the strain differences of E. granulosus that exists in different geographical locations [13].

Sex wise distribution of the parasite indicated that higher rate of hydatidosis was observed in male adult cattle compared to these young cattle and it was also common in local breeds compared to that of cross breed cattle. This might be due to slaughtering of adult male cattle and high number of local breeds in the municipal abattoir as slaughtering of female animals is not recommended.

At the same time hydatid cysts prevalence of each organ at the abattoir survey was found to have unequal distribution, being predominately in the lung and liver with prevalence rate of $70(17.5 \%)$, 
in the lung 65(14.77\%) in liver 2(0.45\%) in heart and 4(0.9\%) kidney respectively. This is explained by the fact that lungs and livers possess the first great capillaries sites encountered by the migrating echinococcus oncosphere (hexacanth embryo) which adopt the portal vein route and primarily negotiate hepatic and pulmonary filtering system sequentially before any other peripheral organ is involved. In addition, the lungs were mostly infected than any other organ this might be due to the fact that ruminants are slaughtered at older age [5].

Hydatid cyst count is highest in the lungs followed by liver and heart. Higher numbers of medium and large sized cysts were found in lungs of cattle than in the liver while the liver harboured higher number of small sized and calcified cysts. The reason for higher percentage of medium and large cysts in the lungs is due to softer consistency of the lung while the higher yield of calcified cysts in liver could be attributed to relatively higher reticulo-endothelial cells and abundant connective tissue reaction of the organ. The high proportion of small cysts may be due to immunological response of the host which might preclude expansion of cyst size [14].

The present finding was also in agreement with Meresie [15], who stated that major organs condemnations in abattoir were occurred due to hydatidosis with greater rejection rates of lungs followed by liver.

Therefore, owing to the presence of socio-economic conditions favourable for hydatidosis and maintenance of high level of infection in the study area and considering the incalculable indirect losses from it, the epidemiology \& control of hydatidosis is often considered to be a duty of veterinary professionals. However, collaboration between veterinarians \& public health workers is essential for successful control of hydatidosis [16].

\section{Conclusions and Recommendations}

Hydatidosis is one of the highly prevalent parasitic diseases of cattle in shire and incurring huge economic loss due to organ condemnation and indirect weight loss. The disease is difficult to control due to backyard slaughtering, lack of adequate meat inspection and habit of raw offal which give for their dogs. The distribution in different organs showed that it was higher in lung and liver compared to the other organs which is responsible for rejection of these edible organs.

- In the view of present findings the following points were recommended;

- Stray dogs control of should be designed and implemented.

- Awareness creation should be given for butchers, abattoir workers and dog owners.

- Preventing of dogs access to raw offal is an effective measure.

- Supervision of slaughtering practices to prevent illegal slaughtering of animals should be in place.

- Proper deworming of dogs is essential

\section{Acknowledgements}

We would like to acknowledge Mekelle University College of veterinary medicine for its overall support during the career of education and also veterinarians working in the shire abattoir for their assistance during data collection.

\section{References}

1. DACA (2006) Standard veterinary treatment guide lines for veterinary practice, Ethiopia.

2. Eckert J1, Deplazes P (2004) Biological, epidemiological, and clinical aspects of echinococcosis, a zoonosis of increasing concern. See comment in PubMed Commons below Clin Microbiol Rev 17: 107-135.

3. Jones O, Kebede N, Kassa T, Tilahun G, Macias C (2012) Occurrence of bovine hydatidosis and evaluation of its risk to humans in traditional communities of Southern Region of Ethiopia. Ethiop. J. Health Dev. 26: 43-48

4. FAO (2004) Food and Agriculture Organization of the United Nations.

5. Macpherson CN, Zeyhle E, Romig T (1984) An Echinococcus pilot control programme for north-west Turkana, Kenya. See comment in PubMed Commons below Ann Trop Med Parasitol 78: 188-192.

6. Radfar MH, Iranyar N (2004) Biochemical profiles of hydatid cyst fluids of E.granulosus of human and animal origin in Iran. Veterinarski Arhir 74: 435-442.

7. Jenkins DJ1, Romig T, Thompson RC (2005) Emergence/re-emergence of Echinococcus spp.--a global update. See comment in PubMed Commons below Int J Parasitol 35: 1205-1219.

8. Lobago F (1994) Echinococcus in Conso (southern Ethiopia). An assessment of its prevalence, economic and public health importance; DVM Thesis, Faculty of Vet. Med. Addis Ababa University.

9. Jobre Y, Labag F, Tirone R, Abebe G, Dorchines P (1996) Hydatidosis in three selected regions in Ethiopia: an assessment trial on its prevalence, economic and public health importance. Rev. Med. Vet, 147: 797-804.

10. Thrusfield M (2007) Veterinary Epidemiology, 3rd ed.Singapore, Black well Science, pp 233

11. Dawit (2008) Prevalence of bovine hydatidosis at Mekelle municipal abattoir DVM thesis, Faculty of veterinary medicine, Alemaya University, Ethiopia.

12. Feseha G (1995) Zoonotic disease in Ethiopia. Ethiopian society of animal production, Addis Ababa.

13. McManus DP (2006) Molecular discrimination of taeniid cestodes. See comment in PubMed Commons below Parasitol Int 55 Suppl: S31-37.

14. Torgerson $P(2002)$ Transmission dynamics of taeniid parasites in animal hosts, Pp. 221-235.

15. Meresie A (1985)A survey of bovine Echinococcosis \& public health significance in Sodo. DVM Thesis, FVM, Addis Ababa University, Ethiopia.

16. Thompson RCA, Allsop CE (1988) Hydatidosis, veterinary perspective and annotesed. Bibliography C.A.B international, Pp 1-16. 